\title{
Dynamic solution to the EEG source localization problem using Kalman Filters and Particle Filters
}

\author{
Javier M. Antelis and Javier Minguez
}

\begin{abstract}
In this paper, we propose a solution to the EEG source localization problem considering its dynamic behavior. We assume a dipolar approach which makes the problem nonlinear. From the dynamic probabilistic model of the problem, we formulate the Extended Kalman Filter and Particle Filter solutions. In order to test the algorithms, we designed an experimental protocol based on error-related potentials. During the experiments, our dynamic solutions have allowed the estimation of sources which are varying in position and moment within the brain volume. Results confirm the activation of the anterior cingulate cortex which is the brain structure associated with error processing. These findings demonstrate the good performance of the dynamic solutions for estimating and tracking EEG neural generators.
\end{abstract}

\section{INTRODUCTION}

Electroencephalography (EEG) is a brain imaging technique that gives a unique access to the electric neural activation, furthermore it has very good temporal resolution, and it is non-invasive, very cheap and portable. Hence, EEG is one of the most preferable technologies to study the brain. However, clinical and functional interpretations of EEG signals imply the speculation of the possible active areas within the brain that generate those signals. For this reason, the solution to the EEG source localization problem attempts to find from EEG signals which regions of the brain are active. There are two general approaches to solve the EEG source localization problem, dipolar methods and distributed methods [1]. Despite their differences, the large majority of these methods share the property of providing static solutions, they only use information from one time instant, whereas the EEG sources and signals clearly have a time varying nature.

To account for this limitation, in this paper we propose a methodology for solving the so-called inverse problem in EEG considering its dynamic nature. We first formulate the dynamic model of the EEG source localization problem in a probabilistic framework. Subsequently, we assume a dipolar approach where sources positions and moments have to be estimated. Under this context we derive the Extended Kalman Filter (EKF) and Particle Filter (PF) solutions, which give the advantage of consider that the neural generators of the EEG signals changes according to some neural dynamic. Additionally, these solutions allow to incorporate in the model the presence of noise in the measurements

J.M. Antelis and J. Minguez are with the I3A and Department of informatics and Systems Engineering, University of Zaragoza, 50018 Zaragoza, Spain.antelis@unizar.es, jminguez@unizar.es

This work has been partly supported by the Spanish project DPI200615630-C02-02 and by grants of Santader Bank and University of Zaragoza and even in the sources. An alternative advantage of PF is that it can explicitly represent simultaneously many likely solutions. In order to validate these formulations, we design a neurophysiological protocol based on error-related potentials where the main focus of neural activity is assumed to be known as reported by other studies. The overall result is that we successfully apply the EKF and PF in real settings to estimate EEG sources.

\section{DYNAMIC MODEL OF THE EEG SOURCE LOCALIZATION PROBLEM}

Let be $\Phi_{0 \ldots t}$ the set of EEG signals up to time $t$ acquired with $N_{e}$ electrodes attached at the scalp. The neural generators of these signals are $N_{s}$ sources. The goal is to estimate the sources parameters $X=\left[r_{1}, J_{1} \ldots r_{N_{s}}, J_{N_{s}}\right]$ (with $r$ being the position and $J$ being the moment) at time $t$ using the measurements up to time $t$. For this, two probabilistic equations are needed, a transition model $p\left(X_{t} \mid X_{t-1}, w_{t}\right)$ and a measurement model $p\left(\Phi_{t} \mid X_{t}, v_{t}\right)$. Where $X_{t}: \Re^{6 \cdot N_{s}}$ is the source space, and $\Phi_{t}: \Re^{N_{e}}$ is the measurement space. The random variables $w_{t}$ and $v_{t}$ represent the noise in the process and in the measurements respectively.

With this representation, the goal is to compute a posterior probability $p\left(X_{t} \mid \Phi_{0 \ldots t}\right)$ to make inference about the sources [2]. Using the Bayes theorem and assuming Markov process we obtain the recursive update equation known as the Bayes filter for the dynamic EEG source localization problem:

$p\left(X_{t} \mid \Phi_{0 \ldots t}\right)=\eta \cdot p\left(\Phi_{t} \mid X_{t}\right) \cdot \int p\left(X_{t} \mid X_{t-1}\right) p\left(X_{t-1} \mid \Phi_{t-1}\right) d X_{t-1}$

Where $\eta$ is a normalizing factor. To implement a solution for the Bayes filter we need three distributions, the initial posterior $p\left(X_{0}\right)$ that characterizes the prior knowledge about the sources, the transition model $p\left(X_{t} \mid X_{t-1}\right)$ that models the time evolution of the neural sources, and the likelihood $p\left(\Phi_{t} \mid X_{t}\right)$ that allows to obtain measurements given a source space. The goal now for solving the dynamic EEG source localization problem is to derive a solution to the Bayes filter.

\section{A. Transition model}

This mathematical model describes how the EEG sources evolve over time which is unknown. Therefore we can assume the transition as a random walk in the source space [2], whereby the specific form of the transition model is $p\left(X_{t} \mid X_{t-1}\right)=\mathcal{N}\left(X_{t} \mid X_{t-1}, Q\right)$. This assumption imposes that the transition model is a zero-mean Gaussian density with a diagonal covariance matrix $Q$ whose elements represent the expected time evolution of each source parameter. 
B. Deriving dynamic solutions to the EEG source localization problem

The dynamic EEG source localization problem can be categorized depending on whether the location of the sources is fixed or not, and on whether the type of the noise in the measurements and in the sources is assumed to be Gaussian or not. We focus on the dipolar approach where the location of the sources is not fixed (non-linear situation) and we assume Gaussian noise. This is because we are interested in modeling few focal areas of the brain. For this case the unknown source space is given by the set of positions and moments for each source $X=\left[r_{1}, J_{1}, \ldots r_{N_{s}}, J_{N_{s}}\right]$, the transition model is given by $\mathcal{N}\left(X_{t} \mid X_{t-1}, Q\right)$ and the measurements model is given by $p\left(\Phi_{t} \mid X_{t}\right)$. We will derive now the EKF and PF dynamic solutions to the EEG source localization problem.

1) Aplying Extended Kalman Filter (EKF) to solve the dynamic EEG source localization problem: The EKF solution assumes that the nonlinear equation of the measurement model can be locally linearized. In order to estimate $X_{t}$ at time $t$, the EKF performs recursively two steps, $i$ ) a time update step which estimates the next state $X_{t}$ using the linear transition equation, and $i i$ ) a measurement update step which adjust the estimated state $X_{t}$ by using the current measurements $\Phi_{t}$ via the linearization of the measurement model [3].

2) Aplying Particle Filters $(P F)$ to solve the dynamic EEG source localization problem: The goal in this solution is to get a set of $N$ samples or particles $\left\{X_{t}^{(i)}\right\}_{i=1}^{N}$ that represents the posterior distribution $p\left(X_{t} \mid \Phi_{0 \ldots t}\right)$. We start with a set of particles $\left\{X_{t-1}^{(i)}\right\}_{i=1}^{N}$ distributed according to $p\left(X_{t-1} \mid \Phi_{0 \ldots t-1}\right)$, then, by applying the transition model to each particle we obtain a new set $\left\{\tilde{X}_{t}{ }^{(i)}\right\}_{i=1}^{N}$ which is distributed according to $p\left(X_{t} \mid \Phi_{0 \ldots t-1}\right)$. Then, using the actual measurements, importance weights $\left\{w_{t}^{(i)}\right\}_{i=1}^{N}$ are computed through the likelihood function $w_{t}^{(i)} \sim p\left(\Phi_{t} \mid \tilde{X}_{t}^{(i)}\right)$. Afterward, a resampling-selection step is applied to the weighted sample set $\left\{\tilde{X}_{t}{ }^{(i)}, w_{t}^{(i)}\right\}_{i=1}^{N}$ discharging/multiplying particles with low/high importance weights [5], so that finally we get the sample set $\left\{X_{t}^{(i)}\right\}_{i=1}^{N}$ distributed according to the posterior $p\left(X_{t} \mid \Phi_{0 \ldots t}\right)$ which can be used to make inference about the sources space $X_{t}$ at time $t$.

3) Parameters of the methods: To implement these nonlinear dynamic solutions, we further need the process covariance $Q$, which is determined based on physiological basis; the noise covariance $R$, which can be estimated with some EEG measurements; the initial estimation $X_{0}$ which can be computed using a static solution such as Beamforming LCMV [4] and the initial density $p\left(X_{0}\right)$ which can be normally distributed with mean $X_{0}$ and covariance $Q$. In the PF, the likelihood function is assumed to be a zero-mean Gaussian function $p\left(\Phi_{t} \mid X_{t}\right)=\mathcal{N}\left(\Phi_{t} \mid X_{t}, R\right)$ and the particle with the highest weight in the posterior is selected to be the sources state $X_{t}$.

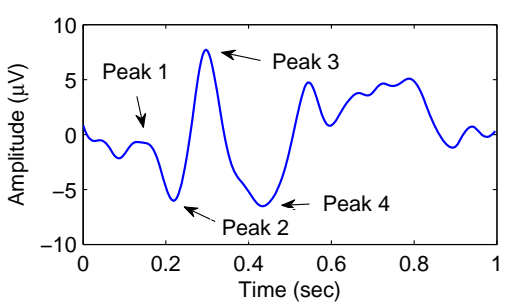

Fig. 1. Typical waveform of an interaction error-related potential at electrode $\mathrm{Cz}$ recorded during the experimental protocol.

\section{EXPERIMENTAL VALIDATION}

\section{A. Experimental protocol, instrumentation and head model}

In order to assess in real settings the performance of the algorithms, we have designed a protocol based on errorrelated potentials (ErrP) since there is evidence of the brain regions that generate these potentials [6].

Recently, it has been probed during brain-computer experiments that an ErrP is evoked after a person is aware of the occurrence of an interaction error [7]. Roughly, this error (named interaction ErrP) has a waveform with four prominent peaks as shown in figure 1. The importance of this ErrP in our context is that for these peaks the main focus of neural activity is expected to be mainly in the anterior cingulate cortex or ACC (Brodmann areas 24 and 32). To elicit the ErrP we have implemented a protocol similar to Ferrez's protocol, where a subject facing a computer screen is concentrated in a green block that is moving from right to left (machine task). While the machine is executing the task, sometimes the block moves to the right (which emulates a machine error) evoking the ErrP. We performed this experiment with one subject while EEG signals were acquired. The whole experiment consisted of 11 sessions with 5 errors trials each one.

The general instrumentation is based on a commercial gTec EEG system. The EEG signals were digitalized with a sampling frequency of $256 \mathrm{~Hz}$, power-line notch-filtered and bandpass-filtered between 0.5 and $60 \mathrm{~Hz}$. After the recording sessions, the EEG signals were average referenced and band pass filtered from 1 to $10 \mathrm{~Hz}$. A time window of one second was selected after the ErrP stimulus onset.

The head was modeled by a homogeneous sphere of radius $96 \mathrm{~mm}$ that represents the skin and the sources were restricted to be located only within a innermost sphere of radius $84 \mathrm{~mm}$ that represents the brain. The measurement space is a set of 32 electrodes from the 10-10 international system projected to lying on the sphere surface. In order to solve the forward model, the head model, the sources position and the electrodes locations where defined in the coordinate system of the MNI head model.

\section{B. Analysis and Results}

For all the ErrP single trials, the main neural sources were estimated using the EKF and the PF. We have also computed the static dipolar solution given by the beamforming LCMV algorithm. 
TABLE I

TWO MORE FREQUENT BRAIN STRUCTURE AND BRODMANN AREA ESTIMATED WITH LCMV, EKF AND PF AT THE OCCURRENCE OF THE PEAKS FOR ALL THE ERRP'S SINGLE TRIALS.

\begin{tabular}{|c|c|c|c|c|c|c|}
\hline Peak & \multicolumn{2}{|l|}{ LCMV } & \multicolumn{2}{|l|}{ EKF } & \multicolumn{2}{|l|}{$\mathrm{PF}$} \\
\hline 1 & $\begin{array}{l}\text { Anterior Cingulate/Gyrus } \\
\text { BA's } 24,25,32,33 \\
\text { Parahippocampal Gyrus } \\
\text { BA's } 27,28,34,35\end{array}$ & $18 \%$ & $\begin{array}{l}\text { Anterior Cingulate } \\
\text { BA's 24, 32 } \\
\text { Cingulate Gyrus } \\
\text { BA's 24, 25, 32, } 33 \\
\end{array}$ & $18 \%$ & $\begin{array}{l}\text { Anterior Cingulate/Gyrus } \\
\text { BA's } 24,25,32,33 \\
\text { Posterior Cingulate } \\
\text { BA's } 23 \text { and } 31\end{array}$ & $\begin{array}{l}73 \% \\
18 \%\end{array}$ \\
\hline 2 & $\begin{array}{c}\text { Parahippocampal Gyrus } \\
\text { BA's } 27,28,34,35 \\
\text { Lingual Gyrus } \\
\text { BA's } 18\end{array}$ & $36 \%$ & $\begin{array}{c}\text { Anterior Cingulate/Gyrus } \\
\text { BA's } 24,25,32,33 \\
\text { Parahippocampal Gyrus } \\
\text { BA's } 27,28,34,35\end{array}$ & $27 \%$ & $\begin{array}{l}\text { Anterior Cingulate/Gyrus } \\
\text { BA's } 24,25,32,33 \\
\text { Parahippocampal Gyrus } \\
\text { BA's } 27,28,34,35\end{array}$ & $\begin{array}{l}55 \% \\
27 \%\end{array}$ \\
\hline 3 & $\begin{array}{c}\text { Anterior Cingulate } \\
\text { BA's } 24,25,32,33 \\
\text { Insula } \\
\text { BA's } 13\end{array}$ & & $\begin{array}{c}\text { Anterior Cingulate/Gyrus } \\
\text { BA's } 23,24,32 \\
\text { Medial Frontal Gyrus } \\
\text { BA's }\end{array}$ & $18 \%$ & $\begin{array}{c}\text { Cingulate Gyrus } \\
\text { BA's 23, 24, 32 } \\
\text { Anterior Cingulate } \\
\text { BA's 24, 32 }\end{array}$ & $\begin{array}{l}36 \% \\
27 \%\end{array}$ \\
\hline 4 & $\begin{array}{c}\text { Parahippocampal Gyrus } \\
\text { BA's } 27,28,34,35 \\
\text { Anterior Cingulate } \\
\text { BA's } 24,32\end{array}$ & $\begin{array}{l}55 \% \\
27 \%\end{array}$ & $\begin{array}{c}\text { Parahippocampal Gyrus } \\
\text { BA's } 27,28,34,35 \\
\text { Anterior Cingulate/Gyrus } \\
\text { BA's } 23,24,32,33\end{array}$ & $36 \%$ & $\begin{array}{c}\text { Parahippocampal Gyrus } \\
\text { BA's } 27,28,34,35 \\
\text { Cingulate Gyrus } \\
\text { BA's } 23,24,32\end{array}$ & $\begin{array}{l}36 \% \\
27 \%\end{array}$ \\
\hline mean $(0.5 \mathrm{sec})$ & $\begin{array}{c}\text { Anterior Cingulate } \\
\text { BA's } 24,32 \\
\text { Cingulate Gyrus } \\
\text { BA's } 23,24,32,33\end{array}$ & $45 \%$ & $\begin{array}{l}\text { Anterior Cingulate } \\
\text { BA's } 24,32 \\
\text { Cingulate Gyrus } \\
\text { BA's } 23,24,32\end{array}$ & $55 \%$ & $\begin{array}{c}\text { Anterior Cingulate } \\
\text { BA's } 24,32 \\
\text { Cingulate Gyrus } \\
\text { BA's } 23,24,32\end{array}$ & $63 \%$ \\
\hline
\end{tabular}

1) Dynamic neural estimation of the ErrP single trials: The objective of this first analysis was to assess the estimations obtained with the EKF, PF and LCMV solutions for all the ErrP's single trials. As in previous studies, we assume 1 dipolar source [6]. Commonly, event related potentials such as ErrP's requires the average of many trials in order to reduce the noise levels, however, our dynamic approaches allow the source localization of ErrP single trials since the algorithms incorporate in the solution model the presence of noise in the measurements. Table I shows the two more frequent brain structures and their associated Brodmann areas estimated by the three methods precisely at the occurrence of the four peaks showed in figure 1. As it can be seen, for the majority of the ErrP peaks, the source is located in the Anterior Cingulate (BA's 24 and 32) and in the Cingulate Gyrus (BA's 25 and 33). These results demonstrates that in the majority of the trials, the ACC is systematically activated during the occurrence of the four prominent peaks. For example, notice that for the first peak the main focus of brain activity estimated by the EKF and PF is located in the Anterior Cingulate and in the Cingulate Gyrus in the $73 \%$ of the ErrP's, however the main focus of activity estimated by the LCMV algorithm is located in these regions in the 55\% of the ErrP's. This table also shows the location of the mean estimated source over half a second after the ErrP stimulus onset. For this case, the EKF and the PF estimated the brain activity in the anterior cingulate (BA's 24 and 32) in 55\% and $63 \%$ of the ErrP's respectively. These findings agree with the mean location over half a second reported in other neurological studies of error potentials [6]. To summarize, these results confirm the fact that at the occurrence of the ErrP peaks and during the whole time interval after that the ErrP is elicited, the main focus of neural activity is located in the brain region covered by the ACC.

2) Neural estimation assuming one and two dipolar sources: In the second analysis the objective was to assess the estimation given by the dynamic solutions during the whole ErrP time window assuming 1 and 2 dipolar sources. Figure 2 shows the position time course during half a second of the estimated sources obtained with the EKF solution over a brain sagittal view. Brodmann areas 24 and 32 are represented by the dark grid within the brain volume. The solution obtained assuming one dipole (figure 2a) shows that during the occurrence of the ErrP peaks the source is located very near to the volume that covers the ACC. Similarly, in the solution obtained assuming two dipoles (figure $2 b$ ) one of the sources (plotted in red) is located almost during the whole time window within the grid that covers the ACC. Comparing the moment of this source with the moment of the second source (plotted in green) during the ErrP peaks, we found that the strength of the source within the ACC is greater than the source outside the ACC. These results explicitly shows that assuming one or two dipoles, the PF estimate the sources within the volumen that cover the ACC.

3) Comparison with static methods: Finally, figure 3 displays for one of the ErrP's the estimated position and moment components obtained with the three methods assuming one dipolar source. Notice that all the methods produce similar estimations. From these results, one could infer that three methods of very different nature (one static method and two dynamic solutions with different hypotheses) estimate closely the same solution which indicates the validity of the proposed dynamic EKF and PF solutions. However, in some time instants, the graph of the estimated position given by the static LCMV method shows abrupt changes. To evaluate this observation, figure 4 shows the magnitude of the position first derivative given by the algorithms. This result explicitly shows the sudden variations in the estimation of the position given by methods that do not address the dynamic nature of the problem. In particular, it can be observed that in many times the position changes abruptly for the static Beamforming LCMV solution, on the other hand the position 


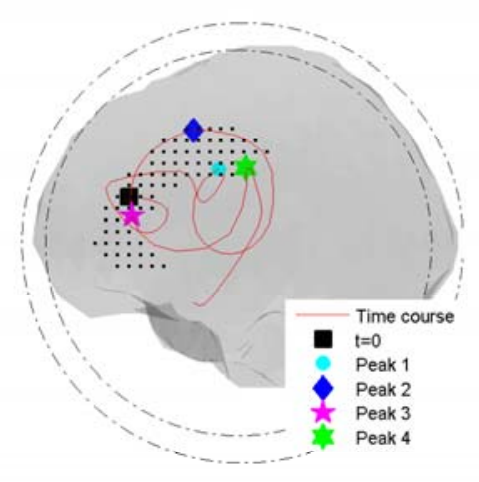

(a)

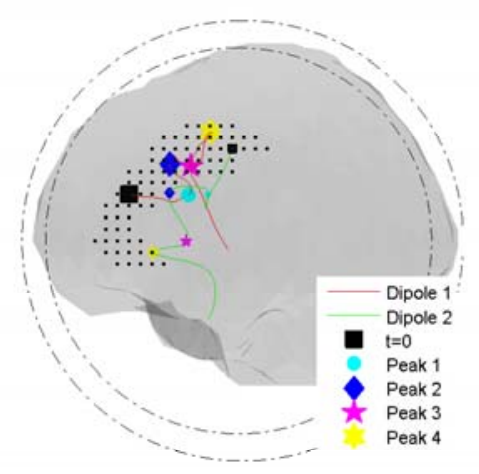

(b)

Fig. 2. Views of the brain showing the estimation time course given by the EKF assuming (a) one dipolar source and (b) two dipolar sources. Dotted circles represent the spherical head model used to solve the forward problem.

always change gradually for the EKF and PF estimations. From these results, we can state that the dynamic methods show a good performance in terms of position estimation since they consider that the dynamic position changes of the EEG sources occurs gradually.

\section{CONCLUSIONS AND FUTURE WORKS}

We have described a methodology to solve the EEG source localization problem considering its dynamic nature. We first obtain the Bayes filter for the EEG source localization problem which allows to recursively update the posterior probability of the neural sources given EEG measurements. Subsequently, for the dipolar and non-linear approach we derived the EKF and PF solutions. To assess the performance of the algorithms we designed a neurophysiological protocol based on error-related potentials. Our dynamic solutions confirm the activation of the ACC during the ErrP peaks which is the brain region associated with error processing. These results demonstrate that the application of EKF and PF for estimating the EEG neural sources is very promising, which can be explained by the fact, among other reasons, that these algorithms take into account the neural dynamics, the non-linearity of the measurement model, and the presence of

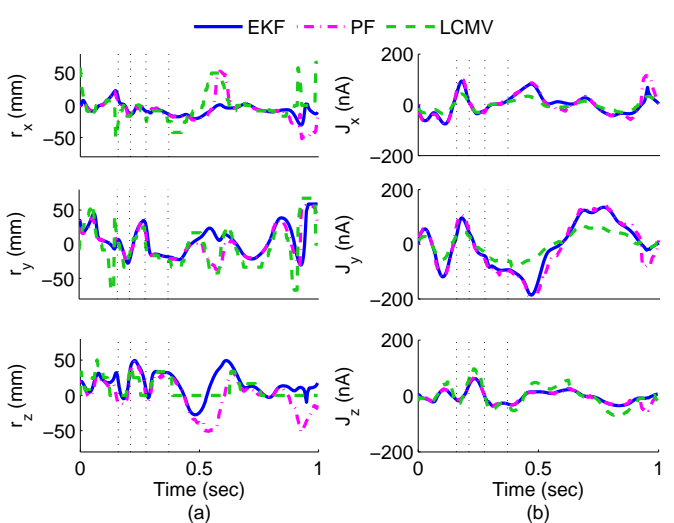

Fig. 3. Time course estimation of the (a) position and (b) moment components for one of the ErrP's trial.

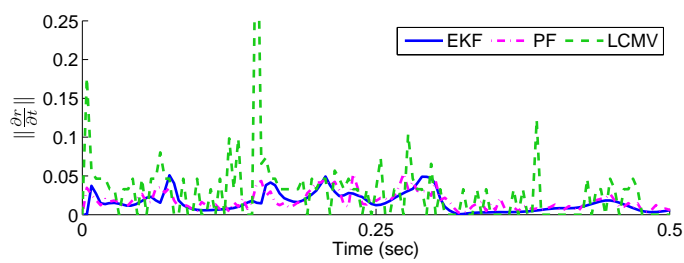

Fig. 4. Magnitude of the position first derivative given for the EKF, PF and LCMV solutions.

noise in the measurements and in the EEG neural generators. In the future work, a more intensive experimental procedure using different kinds of error potentials such as response and observation errors will be used to validate the algorithms. Also, we will extend their implementation in realistic head models. Finally, we will improve the potentiality of the algorithms in three aspects, first, in the EKF the on-line recognition of error during $\mathrm{BCI}$ experiments, in the $\mathrm{PF}$ the identification of various likely solutions, and third, a switching model approach by selecting at each time instant the best solution between various EKF's and PF's running in parallel.

\section{REFERENCES}

[1] R. Grech, T. Cassar, J. Muscat, K. Camilleri, S. Abri, M. Zerbakis, P. Xantho, V. Sakkalis, and B. Vanrumste. Review on solving the inverse problem in EEG source analysis. Journal of NeuroEngineering and Rehabilitation. vol 4, 2008, pp 5-25.

[2] E. Somersalo, A. Voutilainen and J.P. Kaipio. Non-stationary magnetoencephalography by Bayesian filtering of dipole models. Inverse Problems. vol 19, 2003, pp 1047-1063.

[3] G. Welch and G. Bishop. An Introduction to the Kalman Filter. UNCChapel Hill, 2006.

[4] B.D. Van Veen, W. Van Dronglen, M. Yuchtman and A. Suzuki. Localization of brain electrical activity via linearly constrained minimum variance spatial filtering. IEEE Transactions on Biomedical Engineering, vol 44, 1997, pp 867-880.

[5] A. Doucet, N. de Freitas and N. Gordon. Sequential Monte Carlo Methods in Practice, Springer-Verlag, 2001.

[6] H. Van Schie, R. Mars, G.H. Coles and H. Bekkering. Modulation of activity in medial frontal and motor cortices during error observation. Nature Neuroscience. vol 7, 2004, pp 549-554.

[7] P.W. Ferrez and J. del R. Millan. Error-Related EEG Potentials Generated during Simulated Brain-Computer Interaction. IEEE Transaction on Biomedical Engineering. vol 55, 2008, pp 923-929. 